\title{
Results of the 2015 elections to the board of directors
}

\author{
Tim Ebbels ${ }^{1}$
}

(C) Springer Science+Business Media New York 2015

The Metabolomics Society is led by the Board of Directors, comprised of 14 volunteer scientists who coordinate the society's activities. The Board includes two ex officio positions (Editor in Chief of the Society's journal, and the Chair of the Early career Members Network, EMN), plus 12 elected positions. Each director serves a term of 2 years, renewable for a further 2 years by election. The elections are held during July to September each year to choose replacements for those directors coming to the end of their term of office. This year, one director stood for re-election, while three directors are stepping down. The Board would like to thank the out-going directors, Dr. Warwick Dunn, Prof. Oliver Fiehn and Prof. Gary Siuzdak, for their great efforts on behalf of the Society during the years they have served.

This year four Director positions were open for election. I am pleased to announce that the following members were elected to the Board: Dr. Darren Creek, Dr. Sastia Prama Putri, Dr. David Broadhurst and Dr. Krista Zanetti.

Dr. Darren Creek rejoins the board for a second term. He is a National Health and Medical Research Council Career Development Fellow at the Monash Institute of Pharmaceutical Sciences, Parkville, Australia, and his research interests include application of metabolomics to parasitology, microbiology and cancer pharmacology, as well as developing bioinformatics approaches. While on the Board, Darren has served as chair of the important Conferences and Training committee, in particular helping develop a new funding scheme for small conferences and

Tim Ebbels

t.ebbels@imperial.ac.uk

1 Computational and Systems Medicine, Department of Surgery and Cancer, Imperial College London, SW7 2AZ London, UK workshops, as well as serving on the Industry Engagement and Metabolite Identification task groups.

Dr. Sastia Prama Putri joins the Board as a newly elected regular Director. She is an assistant professor at the Department of Biotechnology, Graduate School of Engineering, Osaka University. As the founding chair of the EMN she has been the dynamic force behind many of the EMN activities, such as workshops and networking events during the annual meeting and a series of webinars. As an ex officio Director, she has also served on the Conferences and Training committee and the Strategy task group.

Dr. David Broadhurst is Associate Professor of Applied Biostatistics in the department of Medicine, at the University of Alberta, Canada. Following a PhD in neural networks, he soon became recognized expert in design of experiments, signal processing, biostatistics, and machine learning. With Prof. Douglas Kell at the University of Manchester he helped develop protocols for large-scale clinical metabolomics. In 2009 he moved to Cork University Maternity Hospital, Ireland, to investigate presymptomatic metabolite biomarkers predictive of major pregnancy diseases. His current research at Alberta focuses on the application and optimization of diverse multivariate modelling techniques within the domain of systems-biology. He is passionate about educating the community, especially with regard to design of experiments and rigorous statistical protocols. In particular he is an advocate of formalizing a Society-based accreditation of metabolomics workshops/short courses, and the development of accredited online metabolomics educational media.

Dr. Krista Zanetti is a Program Officer in the Epidemiology and Genomics Research Program (EGRP) at the US National Cancer Institute. Since joining EGRP in 2010, her focus has been building infrastructure and capacity to support metabolomics in large population studies. Most 
recently, she spearheaded efforts to establish the trans-NIH international Consortium of Metabolomics Studies (COMETS) that brought together 24 prospective cohorts and 3 consortia to allow investigators to leverage existing resources and develop methods, tools and protocols for data harmonization, quality control and standardization. During her term, she plans to (a) identify and prioritize challenges in the field, (b) further broaden the reach of the Society by engaging population-based researchers, and (c) develop effective strategies to train the next generation of investigators. Dr Zanetti has already been working with and advising the Early-career Member Network Committee as well as participating in the Strategy task group.

The Board wishes to thank all members who stood for election and would encourage anyone with energy-whether on the Board or not-to support the community by getting involved in the Society's activities, for example by joining one of the Task Groups.

We congratulate the new Directors on their election. They will start their terms on 1 October 2015 and we look forward to working with them to fulfill the Society's mission over the coming years. 